\title{
Quantifying the Initial Spray from Fire Sprinklers
}

\author{
NING REN, ANDREW F. BLUM, YING-HUI ZHENG, CHI DO, and ANDRÉ W. MARSHALL \\ Department of Fire Protection Engineering \\ University of Maryland, College Park, MD, USA
}

\begin{abstract}
The performance of water-based fire suppression systems is governed largely by the spray discharge characteristics associated with the nozzle geometry and injection conditions. In many nozzle configurations such as sprinklers, this initial spray is produced by injecting a water jet onto an orthogonal deflector, resulting in thin, unstable, radially expanding streams. These streams ultimately disintegrate into a complex population of drops forming the spray. The initial spray is generated in distinct stages, which include sheet formation, sheet breakup, and ligament breakup. A Sprinkler Atomization Model (SAM) has been developed based on these physics to predict the initial drop velocity, location, and size based on the nozzle geometry and injection conditions. The initial spray from a simplified yet realistic sprinkler geometry has been quantified through detailed measurements to provide insight into these atomization processes and to evaluate SAM performance. Flow visualization revealed that the deflector produces a continuous radially expanding stream resulting from the flow directed over the tines and a connected underlying orthogonal stream resulting from the flow through the spaces. The measured and predicted breakup locations and drop sizes follow $\mathrm{We}^{-1 / 3}$ scaling laws, previously established by other researchers in similar canonical configurations. However, SAM over predicts the volume median drop diameter by as much as $40 \%$, probably due to the absence of models to characterize the orthogonal stream underlying the radially expanding sheet. This orthogonal stream generated by the spaces was measured to consist of nearly $50 \%$ of the flow and produces smaller drops than the radially expanding sheet. The detailed breakup mechanisms for this stream are currently being characterized to improve fidelity of the atomization model.
\end{abstract}

KEYWORDS: suppression, modeling, sprinkler

\section{NOMENCLATURE LISTING}

\begin{tabular}{|c|c|c|c|}
\hline$A$ & wave amplitude (m) & $W e$ & Weber number \\
\hline$D_{o}$ & effective flow diameter (m) & $\mathrm{z}$ & axial coordinate $(\mathrm{m})$ \\
\hline$d$ & diameter $(m)$ & Greek & \\
\hline$f$ & dimensionless wave amplitude & $\rho$ & density $\left(\mathrm{g} / \mathrm{m}^{3}\right)$ \\
\hline$g$ & gravitational acceleration constant $\left(\mathrm{ms}^{-2}\right)$ & $\lambda$ & wavelength (m) \\
\hline$h$ & measurement elevation $(\mathrm{m})$ & $\mu$ & Liquid viscosity \\
\hline K & $\mathrm{K}$-factor of the sprinkler $\left(\mathrm{Lmin}^{-1} \mathrm{bar}^{-1 / 2}\right)$ & $\delta$ & boundary-layer thickness (m) \\
\hline$n$ & wave number $\left(\mathrm{m}^{-1}\right)$ & & angle between the median streamline \\
\hline$p$ & Injection pressure (bar) & $\alpha$ & and axial direction $z$ \\
\hline$Q$ & flow rate $\left(\mathrm{Lmin}^{-1}\right)$ & & position in curvilinear body-fitted \\
\hline$Q F_{i}$ & overall spray volume fraction & $\xi$ & coordinate \\
\hline$q_{i}^{\prime}$ & $\begin{array}{l}\text { dimensionless linear density of dispersed } \\
\text { volume flow }\end{array}$ & $\begin{array}{c}\beta \\
\text { Subscrip }\end{array}$ & $\begin{array}{l}\text { non-dimensional sheet thickness } \\
\text { ts }\end{array}$ \\
\hline$q_{i}$ & linear volume density & $a$ & air \\
\hline$R$ & characteristic dispersion length scale & crit & break-up condition \\
\hline $\operatorname{Re}$ & Reynolds number & $d$ & deflector \\
\hline$r$ & radial location $(\mathrm{m})$ & drop & drop \\
\hline$r$ & dimensionless radial location & $i$ & $i$ th drop size class \\
\hline$T$ & sheet thickness (m) & j & $j$ th measurement location \\
\hline$t$ & time $(\mathrm{s})$ & l & liquid \\
\hline$U$ & $\begin{array}{l}\text { average velocity of sheet at the edge of } \\
\text { deflector }(\mathrm{m} / \mathrm{s}) \text { magnitude }\end{array}$ & $\begin{array}{l}\operatorname{lig} \\
0\end{array}$ & $\begin{array}{l}\text { ligament } \\
\text { initial or reference condition }\end{array}$ \\
\hline$V F_{i, j}$ & local spray spatial volume fraction & sh & sheet \\
\hline & velocity component $(\mathrm{m} / \mathrm{s})$ & $v 50$ & volume median \\
\hline
\end{tabular}




\section{INTRODUCTION}

Automatic fire sprinklers are accepted as the fire protection system of choice for a wide variety of applications. To support the development of many types of fire protection systems and the design of fire safe environments, modern engineering practices are currently being established like performance-based design, which requires the prediction of fire behavior using physics-based analytical methods and tools. Despite the simplicity of the basic operating principles for fire sprinklers, the complex physics governing water-based suppression including multi-phase transport processes, flame sheet extinction, and extinction of condensed phase reactions, present profound analytical and modeling challenges. Even the physical mechanisms controlling the sprinkler's simple action to generate a dispersed spray are quite complex and do not yield readily to analysis. Yet, as advanced fire protection engineering practices continue to gain popularity, the need to model fire sprinklers for suppression system and even component analysis is inevitable. In this study, the challenge to characterize the initial spray from sprinklers is addressed to advance understanding of the atomization process in fire sprinklers and to develop models for predicting sprinkler spray characteristics.

The atomization process for sprinklers can be described fundamentally by distinct stages, as illustrated and photographed in Fig. 1 (a) and (b), respectively. The injector forms a vertical water jet that impinges upon a deflector. The redirected jet forms a thin, horizontal film traveling along the top of the deflector. Once the film travels past the deflector, it becomes an unconfined, expanding sheet. The sheet expands radially outwards from the deflector becoming increasingly unstable, creating aerodynamic waves. These sinuous waves grow until the sheet begins to breakup at a critical wave amplitude. The sheet disintegrates into ring-like ligaments that are also inherently unstable. Dilatational waves grow on the ligaments until they reach a critical wave amplitude, initiating ligament breakup into even smaller fragments. These fragments will eventually contract to form spherical drops. A more detailed discussion of these atomization processes can be found in Dombrowski [1], Wu [2] and Ren [3].

The key characteristics of fire suppression sprays have been summarized recently in a review paper by Grant and Drysdale [4]. Detailed sprinkler spray measurements have been reported by Dundas [5], Yu [6] and Wendt and Prahl [7]. More recent studies have been conducted by Widmann et al. [8,9], Sheppard [10] and Putorti [11]. These studies characterized drop sizes, volume flux distributions, and velocities produced by a wide variety of sprinkler configurations operating over a range of injection pressures. From this body of work, it is clear that the drop size is reduced with increasing pressure as quantified by the empirical relationship $d_{v 50} / D_{o}=C W e^{-1 / 3}$. Although these studies verified this $W e^{-1 / 3}$ trend more or less, the constant of proportionality varied significantly between sprinkler configurations. The simple scaling law, provides very little insight into the important parameters governing the initial spray from sprinklers suggesting that higher fidelity predictive methods are required to explain the effect of sprinkler geometry on the spray. In order to move beyond empirical relationships to higher fidelity models, a better understanding of the basic atomization mechanisms from sprinklers is required.

Fire sprinklers are based on variations of the essential impinging jet configuration. A focus on this canonical configuration is therefore useful for understanding basic atomization physics relevant to sprinkler atomization. From this perspective, Blum [12] and Ren [3] have conducted experimental and modeling studies providing break-up details, drop size, and dispersion characteristics in the impinging jet configuration operating at sprinkler injection pressures. Even more fundamental studies have been conducted by Villermaux and Clanet [13] in this configuration. They observed drop formation by rim breakup at the sheet as opposed to the ligament breakup mode observed by Dombrowski [1]. However, Blum and Ren observed that the rim breakup mode occurs at lower pressure (or $\mathrm{We}$ ) while the ligament breakup mode occurs at higher pressures as shown in Fig. 1 (b). Blum and Ren observed many similarities between atomization measurements taken in realistic sprinkler configurations and canonical impinging jet configurations confirming that the fundamental atomization mechanisms described in Fig. 1 provides an appropriate basis for the Sprinkler Atomization Model (SAM) [2]. The current study focuses on the development and evaluation of SAM based on comparisons with detailed atomization measurements taken in a simplified yet realistic sprinkler configuration. 


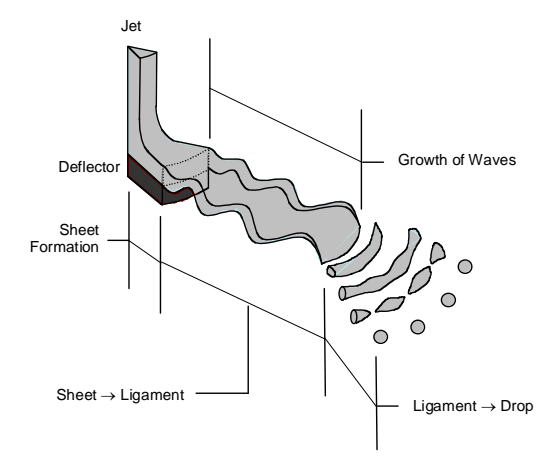

(a)

(b)

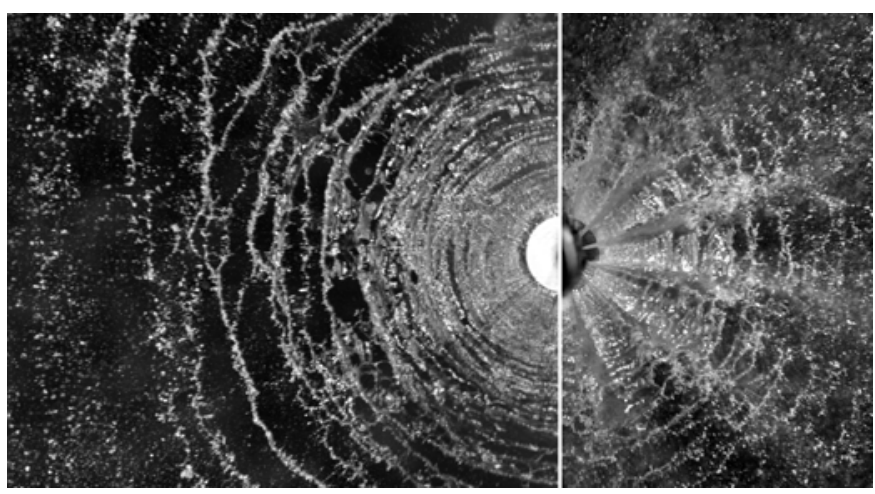

Fig. 1. (a) Illustration of the atomization process [2]; (b) Photograph of the atomization process with $p=$ 2.07 bar for a solid deflector nozzle (left) having geometry similar to the nozzle used in this study (right).

\section{APPROACH}

When dissected, the anatomy of a sprinkler is complex. A thoughtful approach is thus required to evaluate the effect of individual features on spray discharge characteristics. Fig. 2 details the nozzle configuration investigated in this study as well as the important geometric characteristics common to most sprinklers. A commercially available Tyco D3 nozzle was selected to represent a standard sprinkler due to its similarities with conventional sprinklers and its distinct well-characterized geometric features. Table 1 summarizes the important geometric and flow characteristics for all three nozzles used in this study. Detailed measurements were taken at various stages in the atomization region and also within the sprinkler spray to provide insight into the spray formation process, while providing valuable data for evaluation of University of Maryland's Sprinkler Atomization Model (SAM).

\section{Model}

The atomization process in SAM is described with physics based sub-models for sheet formation, sheet trajectory, sheet breakup, and ligament breakup stages. Although first principle multiphase flow models have been established, they are not suitable for large-scale multi-physics engineering level applications. To simplify the complex multi-phase interactions, SAM uses free surface flow theory in the sheet formation and trajectory sub-models, and stability theory for the breakup sub-models. SAM provides the initial velocities, locations, and drop sizes that characterize the spray. After the spray is formed, the problem becomes one of a discrete liquid phase in a continuous gas where spray dispersion can be predicted through Lagrangian tracking models available in codes such as FDS [14]. While a summary of SAM is provided in this section, a detailed description can be found in $\mathrm{Wu}$ et al. [2].

\section{Sheet Formation and Trajectory}

The liquid sheet in sprinklers is formed by impinging a liquid jet onto a deflector. The sheet thickness and velocity are determined from Watson's theory [15] based on a free-surface similarity boundary-layer concept. Following Watson's turbulent theory, when boundary thickness is smaller than the sheet thickness, the sheet thickness is given by

$$
T=\left\{\begin{array}{lc}
D_{0}{ }^{2} / 8 r+0.0245 D_{0}{ }^{1 / 5} r^{4 / 5} / \mathrm{Re}^{1 / 5} & T>\delta \\
1.022 D_{0}{ }^{2} / 8 r+0.0224 r^{5 / 4} /\left(D_{0} R e\right)^{1 / 4} & T<\delta
\end{array}\right.
$$

where $R e=\rho_{l} U D_{o} / \mu_{l}$ is the Reynolds number and the boundary-layer thickness is $\delta=0.303 D_{0}^{1 / 5} r^{4 / 5} / R e^{1 / 5}$. In order to show the effect of viscous interaction with the deflector, a non-dimensional sheet thickness is defined as the actual thickness compared to an inviscid sheet thickness solution, which is given by $\beta=T / T_{0}$ where $T_{0}=D_{0}^{2} / 8 \mathrm{r}$. The non-dimensional form of sheet thickness is the sheet thickening factor, which is

$$
\beta=\left\{\begin{array}{ll}
1+0.196 \operatorname{Re}^{-1 / 5}\left(r / D_{0}\right)^{9 / 5} & T>\delta \\
1.022+0.179 \operatorname{Re}^{-1 / 4}\left(r / D_{0}\right)^{9 / 4} & T<\delta
\end{array} .\right.
$$



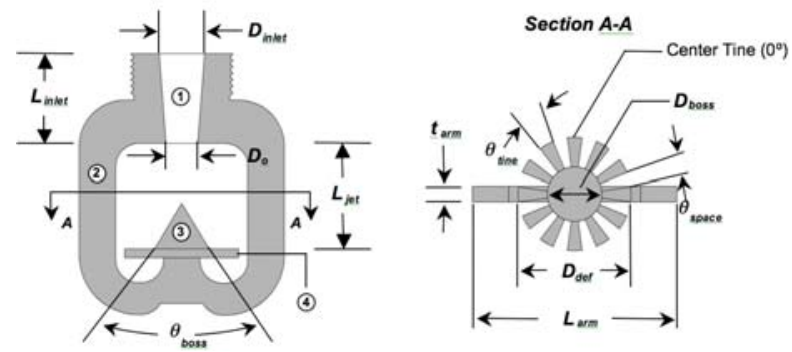

Fig. 2. The anatomy of a sprinkler: (1) Inlet (2) Frame Arms (3) Boss (4) Deflector.

Table 1. Nozzle Dimensions

\begin{tabular}{|c|c|c|c|c|c|c|c|c|c|}
\hline \multicolumn{4}{|c|}{ Inlet } & \multicolumn{3}{|c|}{ Deflector } & \multicolumn{3}{|c|}{ Boss } \\
\hline \begin{tabular}{c}
\hline inlet \\
$(\mathrm{mm})$
\end{tabular} & $\begin{array}{c}L_{\text {inlet }} \\
(\mathrm{mm})\end{array}$ & $\begin{array}{c}L_{\text {jet }} \\
(\mathrm{mm})\end{array}$ & $\begin{array}{c}D_{o} \\
(\mathrm{~mm})\end{array}$ & $\begin{array}{c}\text { K-Factor } \\
\left(\mathrm{lpm} / \text { bar }^{1 / 2}\right)\end{array}$ & $\begin{array}{c}D_{\text {def }} \\
(\mathrm{mm})\end{array}$ & $\begin{array}{c}\theta_{\text {tine }} \\
\left({ }^{\circ}\right)\end{array}$ & $\begin{array}{c}\theta_{\text {space }} \\
\left({ }^{\circ}\right)\end{array}$ & $\begin{array}{c}D_{\text {boss }} \\
(\mathrm{mm})\end{array}$ & $\begin{array}{c}\theta_{\text {boss }} \\
\left({ }^{\circ}\right)\end{array}$ \\
\hline 19.5 & 19.7 & 23 & 6.35 & 25.9 & 25.4 & 20 & 10 & 12 & 65 \\
\hline
\end{tabular}

The average velocity of sheet at the edge of deflector is determined by $U=Q / 2 \pi r_{d} T_{0} \beta$,

where $r_{d}$ is the radius of the deflector. The most important parameter in sheet formation is the sheet thickening factor $\beta$ and average sheet velocity $U$. The sheet velocity governs the wave growth rate and the sheet thickness influences the diameter of the drop. It is also worth noting that the velocity profile of the sheet will have some influence on the sheet break-up. However, in the following analysis, the average sheet velocity is used to simplify the problem.

After leaving the deflector, the external forces acting on the liquid sheet are only the friction force and gravity force. Distinct from a discrete object (i.e. drop), the liquid sheet is a continuous expanding stream, which has a more complex trajectory. Furthermore, the thickness of the sheet changes as the sheet expands radially outwards. Internal forces also affect the trajectory of the sheet especially when the liquid sheet is very thin and the curvature of the trajectory is large. To determine the trajectory of the sheet, a group of differential equations have been developed by Ibrahim [16]:

$$
\left\{\begin{array}{l}
r T \frac{d U}{d \xi}+U T \frac{d r}{d \xi}+U r \frac{d T}{d \xi}=0 ; \quad \frac{d r}{d \xi}=\sin \alpha ; \quad \frac{d z}{d \xi}=\cos \alpha \\
\rho_{l} U \frac{d U}{d \xi}=-\frac{\rho_{a} U^{2}}{2 T}\left[0.79(1+150 T / r) R e_{s h}^{-1 / 4}\right]+\rho_{l} g \cos \alpha \\
\rho_{l} U^{2} \frac{d \alpha}{d \xi}=-\frac{2 \sigma}{T}\left(\frac{\cos \alpha}{r}-\frac{d \alpha}{d \xi}\right)-\rho_{l} g \sin \alpha
\end{array}\right.
$$

The trajectory model is based on curvilinear body-fitted coordinates where $r$ and $z$ are the radial and vertical coordinate of the cylindrical coordinate system. The variable $\xi$ is the position in curvilinear bodyfitted coordinate, $T$ is the local sheet thickness, $U$ is the local sheet velocity, $\alpha$ is the angle between the median streamline and axial direction $z$, and $g$ is the gravitational acceleration constant, $R e_{s h}=2 \rho_{a} r_{d}\left|U_{a}-U\right| / \mu_{a}$.

The initial conditions are specified where the sheet leaves the deflector $(\xi=0)$. The boundary conditions for the sheet velocity and thickness are provided by the impinging jet model. Because there are no good models to predict the initial angle, the initial angle is currently determined empirically. It is not possible to solve the non-linear equations analytically, a fourth order Runge-Kutta method is used to find the solution of $U, T, \theta, r, z$. The purpose of the trajectory model is to predict the local sheet thickness and sheet velocity. In previous atomization studies, the sheet velocity was regarded as a constant by all researchers and the sheet thickness was also treated as a simple function of radius. The trajectory submodel provides those values with more fidelity and accuracy for improved coupling with the sheet break-up model (increases sheet thickness by $3 \%$ to $9 \%$ at breakup). 


\section{Breakup}

As shown in Fig. 1, the sheet formed by the sprinkler is inherently unstable resulting in the growth of aerodynamic waves, which cause the sheet to break. The wavelength at sheet break-up governs the resulting fragment size and ultimately the ligament diameter. A theory based on linear stability has been developed by Dombrowski [1] to predict the wave instability for two dimensional waves in an inviscid gas. In this model, sinusoidal waves are assumed to exist on the liquid sheet. A force balance is performed on the sheet considering inertial, pressure, viscous, and surface tension forces associated with the wave displacement. After considerable reformulation and simplification, the force balance can be expressed in terms of the growth rate of the waves present on the liquid sheet

$\left(\frac{\partial f}{\partial t}\right)^{2}+\frac{\mu_{l}}{\rho_{l}} n^{2}\left(\frac{\partial f}{\partial t}\right)-\frac{2\left(\rho_{a} n U^{2}-\sigma n^{2}\right)}{\rho_{l} T}=0$,

where $t$ is time, $f$ is a dimensionless wave amplitude defined by $f=\ln \left(A / A_{0}\right), A$ is the wave amplitude and $A_{0}$ is initial wave amplitude, $\mu_{l}$ is the liquid viscosity, $T$ is sheet thickness, $n$ is wave number defined by $n=2 \pi / \lambda$, and $\lambda$ is wavelength. The sheet velocity, $U$, and sheet thickness, $T$, is given by the trajectory model. The trajectory and sheet break-up analysis are only weakly coupled. According to the linear wave dispersion theory, the wave amplitude is small compared to the wavelength. The effect of waves on the sheet trajectory can be neglected. However, the sheet thickness and velocity significantly affect the wave growth rate. From the wave dispersion equation, it is apparent that the pressure (or inertial) force accelerates the wave growth while the surface tension attenuates the waves. As the density ratio, $\rho_{a} / \rho_{t}$ increases, the wave growth rate also increases. In real fire scenarios, the gas temperature increases and the density decreases. As a result, the wave growth rate will decrease. Although the wave number, $n$, can be any real number, there is only one wave number that makes the wave grow the fastest. This critical wave number, $n_{\text {crit }}$, is considered to be the most unstable wave that will first lead to breakup. The sheet won't breakup until $f$ reaches the critical dimensionless wave amplitude $f_{\text {crits. }}$. In Dombrowski's theory, $f_{\text {crisst }}$ is a constant with a value of 12 regardless of working conditions. Other researchers also found that $f_{\text {critsh }}$ is a constant, which is close to 12 . It should be noted that in our experiment, $f_{\text {critsh }}$ was assumed to be a function of nozzle configuration and could be determined by experiment. The break-up time is recorded when $f$ reaches $f_{\text {critsh }}$. From the trajectory model, the corresponding sheet velocity, sheet thickness, and the breakup location $r_{\text {crists }}$ are found according to the breakup time.

The thin flapping sheet is assumed to break up into ring-like fragments having a radial extent of one half wavelength. The ligament diameter can be determined from conservation of fragment mass. The ligament diameter is not only related to the ligament mass, but also related to the sheet break-up location. The sheet break-up analysis reveals that the critical wave number and sheet break-up location are important quantities governing atomization behavior. In this study, the sheet break-up location is carefully measured for evaluation of the atomization model. The ligaments produced by the sheet are also unstable. Different from the sheet break-up model, the surface tension force plays a positive role for the wave growth. Weber [17] provided an analysis (similar to the sheet breakup analysis) where the critical wave number is given by

$n_{\text {cirt }, \text { lig }} d_{\text {lig }}=\left[\frac{1}{2}+\frac{3 \mu_{l}}{2\left(\rho_{l} d_{\text {lig }} \sigma\right)^{1 / 2}}\right]^{1 / 2}$.

The unstable waves on the ligament are dilational waves, which will lead to break-up every one wavelength. From conservation of mass, the droplet diameter can be expressed as

$d=d_{\text {lig }}^{2 / 3}\left(3 \pi / n_{\text {crit lig }}\right)^{1 / 3}$.

The ligament breakup time related to the ligament diameter is

$t_{\text {crit }, \text { lig }}=24\left(\frac{2 \rho_{l}}{\sigma}\right)^{1 / 2}\left(\frac{d_{\text {lig }}}{2}\right)^{3 / 2}$.

and the initial drop locations are estimated by $r_{d r o p}=r_{b u, s h}+U_{\text {lig }} t_{\text {crit tig }}$.

The important physics and associated governing equations have been summarized. SAM provides both deterministic and stochastic formulations for predicting the initial spray based on these governing equations. The deterministic formulation provides characteristic values for the initial drop velocity, 
location, and size; however, fire suppression sprays show strong stochastic behavior. For example, the sheet does not always breakup at the same distance and the drops do not have only one diameter. In order to model these stochastic behaviors, probability distributions are introduced into the model to treat the various stages of the breakup process. The sheet critical breakup amplitude, the sheet breakup wavelength, and the ligament breakup wavelength are all treated stochastically. This physics-based technique provides an alternative to specifying a standard distribution about a calculated characteristic drop size. The stochastic model ultimately provides distributions for initial drop velocity, size, and location. A detailed discussion on the stochastic model is provided by $\mathrm{Wu}[2]$.

\section{Measurements}

Sheet breakup, volume density, and local drop size experiments were conducted to quantify discharge characteristics for the representative nozzle. The experiments were performed at $0.69,1.38,2.07$, and 2.76 bar to investigate the effect of injection pressure on spray characteristics.

\section{Flow Visualization}

Photographic and Planar Laser Induced Fluorescence (PLIF) techniques were used in this study to visualize the breakup process and measure sheet/ligament breakup locations. These experiments were conducted inside a vented $1.7 \mathrm{~m} \times 1.7 \mathrm{~m} \times 1.9 \mathrm{~m}$ chamber illustrated in Fig. 3. Using a purely photographic method, the flow was illuminated with two synchronized Canon EX Series flashes reflected from above and orthogonal to the radially expanding sheet, and having discharge times of $15.6 \mu \mathrm{s}$. A Canon 40D 10.1 MP Digital SLR camera was placed above the nozzle to photograph the sheet breakup producing images similar to the one depicted in Fig. 1. At least 20 images were recorded at each experimental condition. Breakup locations were obtained in each image at 37 equally spaced circumferential stations between $-45^{\circ}$ and $45^{\circ}$ and relative to the centerline of a reference tine. Planar Laser Induced Fluorescence was utilized to visualize a cross-section of the expanding sheet. Illumination was provided by a $500 \mathrm{~mW}$, air cooled, argon ion laser and a 20 face rotating mirror spinning at $20 \mathrm{~Hz}$. The water supply was seeded with Rhodamine $6 \mathrm{G}$ dye having a mass concentration of $0.5 \mathrm{mg} / \mathrm{l}$. The sheet was imaged with a low noise, 16bit, 2.0 MP, Cooke SE $\odot$ high-speed digital video camera fitted with a high pass optical filter operated with an electronic shutter speed of $900 \mu$ s at 5 frames per second. These planar images provided insight into the structure of the continuous stream before breakup and the liquid fragments during the breakup process.

\section{Flow Split}

The fraction of the total flow divided between the radial expanding sheet (generated by flow along the tines) and the downward directed streams (generated by flow through the spaces) is measured with two concentric $38 \mathrm{~L}$ and $144 \mathrm{~L}$ containers. The inner container collects the flow through the spaces and the outer container collects the radially expanding flow from the tines as shown in Fig. 3 (b). The opening in the lid of the small container is designed to fit the size and shape of the sprinkler deflector exactly, providing complete separation between the space and tine generated flow streams.
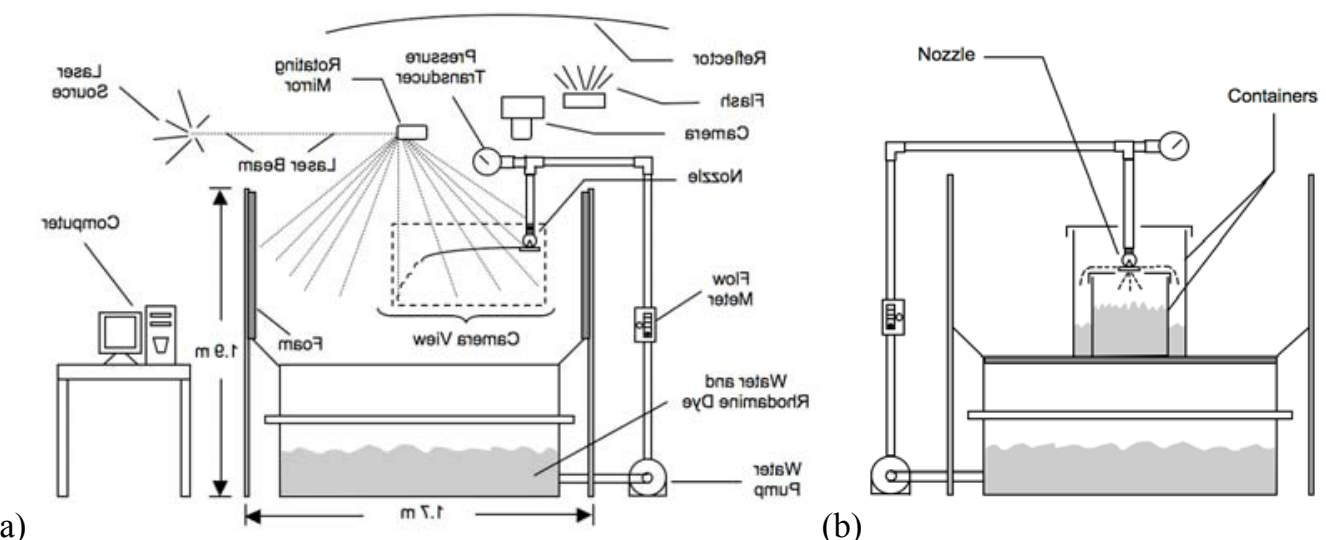

(b)

Fig. 3. (a) Flow visualization experimental set-up; (b) Flow-split experimental setup. 


\section{Dispersion}

Volume density distributions were obtained using a $2.6 \mathrm{~m}$ patternator positioned $1 \mathrm{~m}$ below the nozzle deflector surface and $1 \mathrm{~m}$ above the floor. To permit analysis of the entire sprinkler spray, volume density measurements were conducted inside a large $8.6 \mathrm{~m} \times 7.2 \mathrm{~m} \times 3 \mathrm{~m}$ room located at the Maryland Fire Rescue Institute, as illustrated in Fig. 4. The nozzles were discharged for 10 minutes to average over short time scale aerodynamic or water supply fluctuations, after which the water in each cup was weighed to determine the volume at each radial station. Volume density distributions were aligned with the middle of the center tine $\left(0^{\circ}\right.$ station $)$ and the adjacent space $\left(15^{\circ}\right.$ station $)$.

A characteristic dispersion length scale, $R$, first introduced by Wendt and Prahl [7], was employed to facilitate analysis of the measurements. This reference quantity provides an inviscid radial location at the measurement elevation for each experimental condition, and is given by

$$
R=\left(v_{o}\right)_{r}\left(\frac{2 h}{g}\right)^{1 / 2}\left[\left(1+\frac{\left(v_{o}\right)_{z}{ }^{2}}{2 g h}\right)^{1 / 2}-\left(\frac{\left(v_{o}\right)_{z}{ }^{2}}{2 g h}\right)^{1 / 2}\right],
$$

where $h$ is the measurement elevation (below the nozzle), $g$ is the gravitational constant, $\left(v_{o}\right)_{r}$ is the initial radial sheet velocity, and $\left(v_{0}\right)_{z}$ is the initial axial sheet velocity. The velocity magnitude is estimated from the model described in $\$ 2.1 .1$ and the angle is determined empirically from flow visualization experiments, yielding sheet velocities $\left(v_{o}\right)_{r}$ and $\left(v_{o}\right)_{z}$. The resulting volume density distributions in the $r / R$ coordinate describe the relative effect of drag on dispersion. The volume density measurements were described nondimensionally so that $\sum q_{j}^{\prime} \Delta r^{\prime}=1$, where $\Delta r^{\prime}=\Delta r / R$ is the dimensionless station width and the dimensionless linear density of dispersed volume flow, $q_{i}^{\prime}$, is given by

$$
q_{j}^{\prime}=\frac{q_{j}}{Q / \pi R^{2}}\left(2 r^{\prime}\right)
$$

where $q_{j}$ is the linear volume density measured at measurement location $j$ and $Q$ is the flow rate. The length scale $R$ was modified at every experimental condition for the measurements aligned with the tine $\left(0^{\circ}\right)$. For measurements aligned with the space, the adjacent tine-aligned $R$ values were used for the reference length scale.

\section{Drop Size}

Local drop size measurements were also conducted inside the large room illustrated in Fig. 4 to investigate the drop size variations along the radial span of the spray. An overall drop size distribution and a characteristic drop size, $d_{v 50}$, for each experimental condition can be derived from these measurements. The local drop sizes were measured using a Spraytec spray particle analyzer developed by Malvern Instruments. This laser-based instrument employs a light diffraction technique for counting and sizing drops or particles as illustrated in Fig. 4. Local measurements were taken at 12 radial stations starting at $0.5 \mathrm{~m}$ and separated by $0.5 \mathrm{~m}$, positioned $1 \mathrm{~m}$ below the nozzle and $1 \mathrm{~m}$ above the floor. The Spraytec

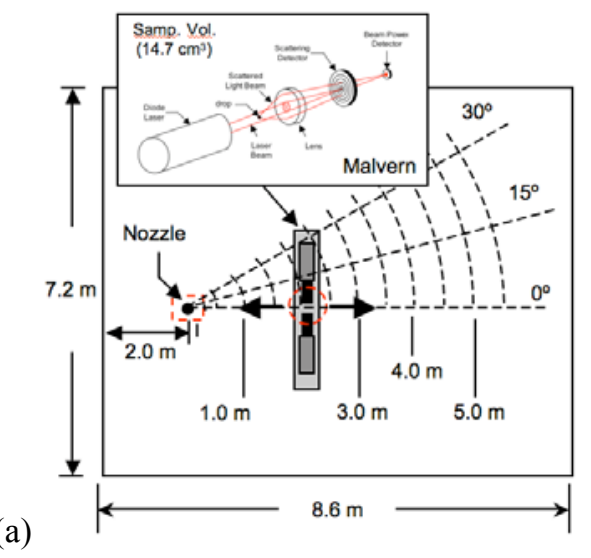

(b)

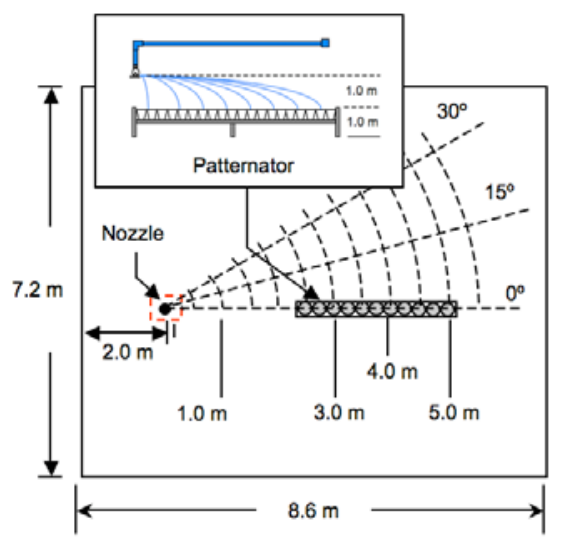

Fig. 4. (a) Drop size measurement experimental set-up; (b) volume flux distribution experimental set-up. 
measurement volume was configured to be $12 \mathrm{~mm}$ in diameter and $130 \mathrm{~mm}$ long. At least 100 drops were estimated to fill the measurement volume at any given station. Measurements were taken at each station for 1 minute at $50 \mathrm{~Hz}$ providing local drop size distribution realizations. The drop sizes were measured at the same circumferential stations as the volume flux experiments. The drop size distribution determined by the Malvern RTSizer software is a local drop size distribution within the Spraytec's measurement volume. However, in this study an overall characteristic drop size, $d_{v 50}$, for the entire spray is of interest. To determine the overall $d_{v 50}$ the local Spraytec measurement is weighted with the local volume density measurements to transform the spatial Malvern measurements into the flux-based drop size distributions described by

$Q F_{i}=Q_{i} / Q_{T} ; Q_{i}=\sum_{j=1}^{N}\left(q_{j}^{\prime}\right) \Delta r^{\prime} V F_{i, j} ; Q_{T}=\sum_{i=1}^{M} \sum_{j=1}^{N}\left(q_{j}^{\prime}\right) \Delta r^{\prime} V F_{i, j} ;$

where $V F_{i, j}$ is the local spatial volume fraction within the Spraytec measurement volume for the $i$ th drop size at the $j$ th measurement location. The spray quantities $Q_{i}$ and $Q_{r}$ are estimates of the drop-wise volume flux and total volume flux from all drops, respectively. The quantity $Q F_{i}$ is the flux-based dropwise volume flux fraction for the entire spray. Drop size distributions based on 60 drop size bins ranging from $0.29-2000 \mu \mathrm{m}$ are easily calculated from $Q F_{i}$ for determining flux based drop characteristics.

\section{RESULTS}

Flow visualization, breakup characterization, drop size measurements along with atomization modeling provided insight into the discharge characteristics of a simple yet realistic sprinkler geometry operated over a range of operating pressures. The nozzle geometry detailed in Fig. 2 and Table 1 was operated at 0.69, 1.38, 2.07, and 2.76 bar. The results from the combined measurements and physics based atomization model are analyzed to help explain the connection between the measured spray behavior and the wellcharacterized nozzle geometry.

Flow visualization revealed the structure of the thin sheet created by the nozzle. A radially expanding sheet is formed from the jet impinging on the tined deflector used in this study as shown in the right most image presented in Fig. 1 (b). This sheet disintegrates into ring-like ligaments similar to the reference sheet created from the solid deflector (having $D_{\text {def }}=25 \mathrm{~mm}$ and $L_{j e t}=75 \mathrm{~mm}$ ) shown on the left in the same figure. However, the sheet structure generated in the realistic tined configuration breaks up earlier than the sheet generated in the canonical configuration and clearly has a more complex three dimensional structure. Although the sheet is continuous, despite the presence of the spaces in this configuration, there appears to be an underlying flow stream aligned with the spaces. This underlying flow stream is deliberately imaged out of focus using a narrow depth of field to better visualize the continuous radially expanding sheet in the overhead images shown in Fig. 1 (b). The underlying stream is better represented in the Fig. 5 PLIF images obtained orthogonal to the central tine $\left(0^{\circ}\right)$ at various distances from the centerline. These planar images show flow through the spaces in the $12.7 \mathrm{~mm}$ plane just at the edge of the deflector. Perhaps, the development of an underlying orthogonal sheet structure is most clearly imaged in the $22.7 \mathrm{~mm}$ plane. By $62.7 \mathrm{~mm}$ the orthogonal sheet appears to have disintegrated completely; however, remnants of the radially expanding sheets aligned with the tines appear to persist. An atomization model has yet to be developed describing disintegration of the flow stream generated by the spaces. To support model developed, more detailed visualization is currently being conducted to capture the important atomization mechanisms in this flow stream.

Determination of the flow split between the radially expanding sheet created from the tines and the orthogonal flow created with the spaces is crucial, not only to understand the contribution of the orthogonal stream to the overall spray, but also to determine the sheet thinning impact from flow divergence through the spaces. This sheet thinning effect is accounted for in Eq. 3. For the nozzle used in this study $51 \%$ of the flow was directed into the radially expanding sheet and approximately $49 \%$ of the flow was directed into the orthogonal stream (virtually independent of $p$ ), which would result in a significantly thinner sheet than that generated in the canonical configuration and explain the earlier sheet breakup.

Detailed analysis of the flow visualization images provides quantitative data for evaluating modeling results at intermediate stages of the atomization process. Consistent with the images provided in Fig. 1 (b), measurements and modeling results are presented in Fig. 6 showing that drops do not initiate at the deflector edge, but instead are formed at some distance beyond the deflector depending on the operating 


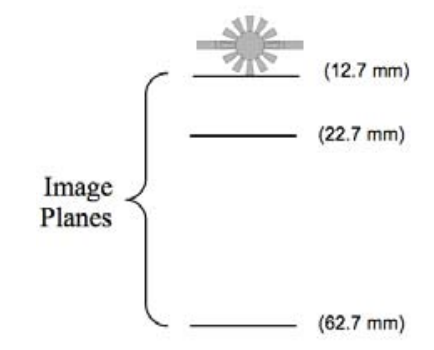

(a)
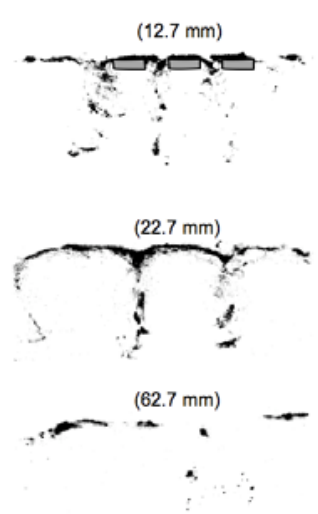

(b)

Fig. 5. Inverted PLIF images depicting flow through sprinkler spaces; (a) top view of measurement locations; (b) three-dimensional stream generated with a tined deflector.

conditions. In fact, even after the sheet breaks up, ligament disintegration must be complete before drops are formed. It is only after disintegration is complete (i.e. drops are formed) that discrete drops should be introduced into the continuous flow domain for CFD analysis. The measured sheet and breakup location distributions at $p=2.07$ bar presented in Fig. 6 (a) show that the breakup process is indeed stochastic having Gaussian-like distributions. In fact, these distributions demonstrate that sheet breakup and drop formation events frequently overlap following visual observations from images.

The mean sheet and ligament breakup results showing the effect of injection condition are provided in Fig. 6 (b). The $W e^{-1 / 3}$ scaling law first observed by Huang [18] in opposing impinging jets in the high $W e$ regime is also observed in this more complex sprinkler configuration. The results in this study show that the sheet breakup and ligament breakup locations follow this scaling law. At very high We (i.e injection pressure), the agreement between the modeled and measured sheet breakup locations is excellent; however, at low pressure the scaling law does not apply. This breakup location deviation is consistent with observations made from flow visualization showing that at low pressure the atomization behavior changes from a 'sheet to ligament' breakup mode to one where the sheet breaks up directly to form drops. The divergent low-pressure behavior is consistent with sprinkler operating recommendations where low pressure injection is avoided to ensure a well-defined spray.

The measured drop size distribution taken at the $0^{\circ}$ station aligned with the tine at $p=2.07$ bar is provided in Fig. 7 (a). The measured drop size distribution follows a log-normal Rosin-Rammler distribution with $d_{v 50}=393$ and $\gamma=2.0$. The measured distribution shows that the drop sizes range from $70 \mu \mathrm{m}$ to $2 \mathrm{~mm}$. The drop size model performance is evaluated through comparing the dashed predicted CVF curve and the solid modeled CVF curve. The shape of the modeled and measured CVF distributions is very close; however, the model is unable to predict the small drops created by the sprinkler configuration with its distribution starting at $200 \mu \mathrm{m}$. The measured small drops may result from the orthogonal stream or another breakup mechanism not accounted for in SAM. Drop size measurements at a variety of injection pressures are plotted against $W e$ in Figure 7 (b). Measurements at the space $\left(15^{\circ}\right)$ and tine $\left(0^{\circ}\right)$ measurement stations appear to follow the $W e^{-1 / 3}$ scaling law at sufficiently high operating pressures. It is also clear from the measurements that orthogonal space stream produces a distinctly different and smaller drop size than the radially expanding sheet formed from the tines.

At first blush, the stochastic model shows a significant over-prediction of the measured drop size. The deviation is expected when comparing the predictions and the measurements aligned with the space, because the model does not account for orthogonal sheet breakup mechanisms. However, the model is based on sheet breakup mechanisms and accounts for the significant loss of flow from the sheet into the orthogonal space stream. Considering the fidelity included in the model, better agreement was expected and this modeling discrepancy deserves closer examination. To understand this discrepancy, the significant difference between drop sizes measured at the station aligned with the center of the tine $\left(0^{\circ}\right)$ and the station aligned with the narrow stream generated through the space $\left(15^{\circ}\right)$ should be recognized. Next it should be 


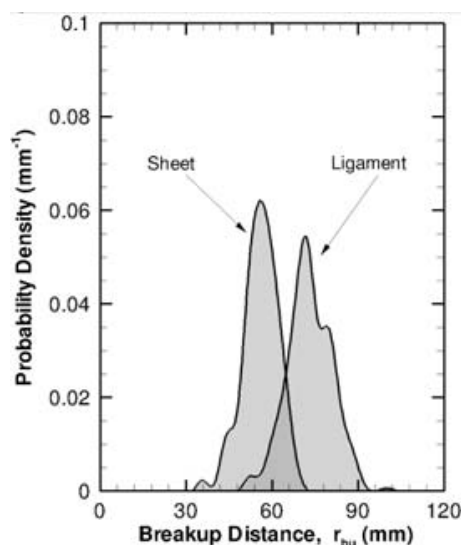

(a)

Fig. 6. (a) Breakup distance distributions for the sheet and ligaments ( $p=2.07 \mathrm{bar}$ );

(b) Breakup distances at various injection pressures (or $\mathrm{We}$ ).

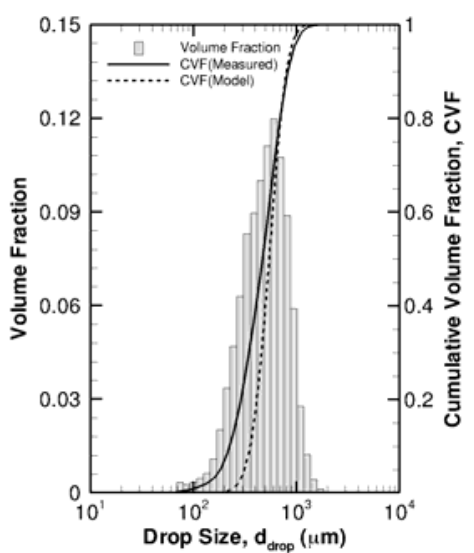

(a)

(a)

(b)

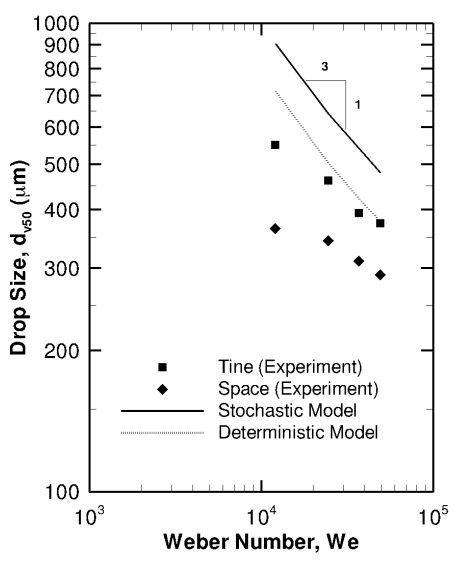

Fig. 7. Drop size measurements and predictions; (a) $0^{\circ}$ station distribution at 2.07 bar;

(b) volume median drop diameter at various injection pressures (or $\mathrm{We}$ ).

noted that just over $50 \%$ of the flow is directed into the sheet, while almost $50 \%$ of the flow forms the orthogonal stream produced by the spaces. Although the two measurements were aligned with the center of the tine and space generated streams, the drops from these streams may be dispersed widely even reaching the adjacent measurement stations $1 \mathrm{~m}$ below the deflector. This dispersion could result in contamination of the drop size measurements and prevent definitive measurement of the distinct initial drop sizes originating from the streams. The mixing of the sheet and space generated drops at their respective measurement stations would bias the sheet generated drop sizes downward and the space generated drop sizes upward. Indeed the volume density measurements provided in Fig. 8 reveal this mixing effect. Fig. 8 (a) shows a strong volume density peak close to the nozzle centerline at the $15^{\circ}$ station aligned with the space. The high volume density near the centerline is consistent with expectations from this configuration, where flow from the jet is forced downward through the spaces by the boss. In contrast, the volume density at the $0^{\circ}$ station aligned with the tine shows distinctive inner and outer peaks at all operating pressures. It becomes apparent from comparison of the distributions at the $0^{\circ}$ and $15^{\circ}$ stations that these two distinctive peaks result from drops generated by the inner directed space flow streams and the outer directed tine generated streams verifying the previous dispersion, mixing and biasing arguments. The small secondary peaks observed at the $15^{\circ}$ station also appear to result from this dispersion effect. With this bias in mind, it follows that SAM predictions from the stochastic model (based purely on sheet breakup physics) would produce larger drop sizes than the measurements even for the station aligned with the tine generated stream. Furthermore, it appears that the good agreement between the lower fidelity deterministic model and the measurements aligned with the tine is purely fortuitous as the deterministic model is missing models not 


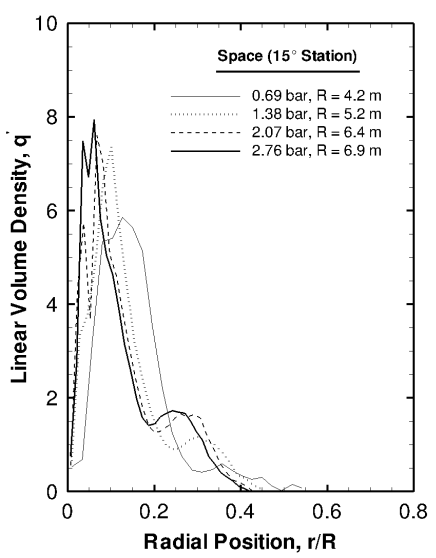

(a)

Fig. 8. Linear volume density for all injection pressures (or $\mathrm{We}$ );

(a) $15^{\circ}$ station aligned with space; (b) $0^{\circ}$ station aligned with tine.

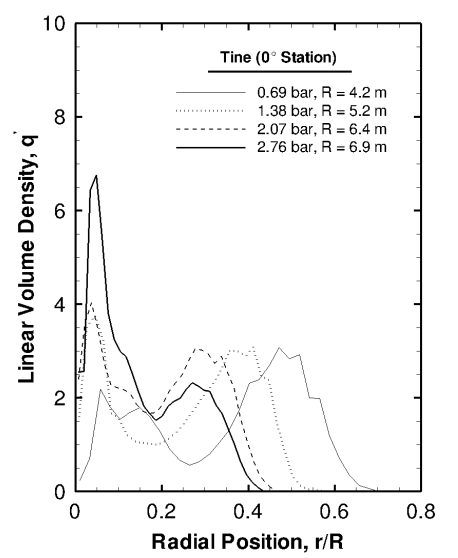

(b) 


\section{REFERENCES}

[1] Dombrowski, N. and Johns, W. R., "The Aerodynamics Instability and Disintegration of Viscous Liquid Sheets", Chemical Engineering Science, Vol.18, pp.203-214, 1963, doi:10.1016/00092509(63)85005-8.

[2] Wu, D, Guillemin, D., and Marshall, A. W., "A Modeling Basis for Predicting the Initial Sprinkler Spray”, Fire Safety Journal, Vol. 42, pp. 283-294, 2007, doi:10.1016/j.firesaf.2006.11.007.

[3] Ren, N.., "Analysis of the Initial Spray from Canonical Fire Suppression Nozzles", M.S. Thesis, University of Maryland - College Park, 2007.

[4] Grant, G., Brenton, J., and Drysdale, D., "Fire Suppression by Water Sprays," Progress in Energy and Combustion Science, Vol. 26, pp. 79-130, 2000, doi:10.1016/S0360-1285(99)00012-X.

[5] Dundas, P. H., "Technical Report Optimization of Sprinkler Fire Protection The Scaling of Sprinkler Discharge: Prediction of Drop Size," FMRC Serial No. 18792 RC73-T-40, Factory Mutual Research Corporation, Norwood, MA, June 1974.

[6] Yu, H. Z., "Investigation of Spray Patterns of Selected Sprinklers with the FMRC Drop Size Measuring System," First International Symposium on Fire Safety Science, New York, pp. 11651176, 1986.

[7] Prahl, J. M., Wendt, B., "Discharge Distribution Performance for an Axisymmetric Model of a Fire Sprinkler Head," Fire Safety Journal, Vol. 14, pp. 101-111, 1988, doi:10.1016/03797112(88)90048-3.

[8] Widmann, J. F., "Phase Droppler Interferometry Measurements in Water Sprays Produced by Residential Fire Sprinklers", Fire Safety Journal, Vol. 36, pp. 545-567, 2001, doi:10.1016/S03797112(01)00009-1.

[9] Widmann, J. F., Sheppard, D. T., and Lueptow, R. M., "Non-Intrusive Measurements in Fire Sprinkler Sprays", Fire Technology, Vol. 37, pp. 297-315, 2001, doi:10.1023/A:1012727614590.

[10] Sheppard, D. T., "Spray Characteristic of Fire Sprinklers," NIST GCR 02-838, National Institute of Standards and Technology, Gaithersburg, MD, 2002.

[11] Putorti, A. D., Everest, D., and Atreya, A., "Simultaneous Measurements of Drop Size and Velocity in Large-Scale Sprinkler Flows Using Particle Tracking and Laser-Induced Fluorescence," National Institute of Standards and Technology, Gaithersburg, MD, 2003.

[12] Blum, A. F., "Discharge Characteristics of Canonical Sprinkler Sprays," M.S. Thesis, University of Maryland - College Park, 2007.

[13] Villermaux, E., Clanet, C., "Life of a Flapping Liquid Sheet", Journal of Fluid Mechanics, Vol. 462, pp. 341-363, 2002, doi:10.1017/S0022112002008376.

[14] McGrattan, K., "Fire Dynamics Simulator (Version 4) Technical Reference Guide", National Institute of Standards and Technology, Gaithersberg, MD, NIST Special Publication 1018, 2004.

[15] Watson, E. J., "The Radial Spread of a Liquid Jet Over a Horizontal Plane", Journal of Fluid Mechanics, Vol. 20, pp. 481-499, 1964, doi:10.1017/S0022112064001367.

[16] Ibrahim, E.A., McKinney, T.R., "Injection Characteristics of Non-swirling and Swirling Annual Liquid Sheets", Jannaf Propulsion Conference, Las Vegas, Nevada, May 10-13, 2004.

[17] Weber, Z., Angew Math Mech., Vol. 11, pp. 136-154, 1931, doi:10.1002/zamm.19310110207.

[18] Huang, J. C., "The Break-up of Axisymmetric Liquid Sheets", Journal of Fluid Mechanics. Vol. 43, part 2, pp. 305-319, 1970, doi:10.1017/S0022112070002392. 